\section{Prevalência e fatores associados ao transtorno da conduta entre adolescentes: um estudo de base populacional}

\author{
Prevalence and factors associated with behavioral \\ disorders in adolescents: a population-based study
}

\footnotetext{
${ }^{1}$ Escola de Psicologia, Universidade Católica de Pelotas, Pelotas, Brasil. 2 Programa de Pós-graduação em Epidemiologia Universidade Federal de Pelotas, Pelotas, Brasil.

Correspondência A. L. S. Cruzeiro Programa de Pós-graduação em Saúde e Comportamento Escola de Psicologia, Universidade Católica de Pelotas.

Rua Almirante Barroso 1202, sala G 109, Pelotas, RS 96010-208, Brasil. alcruzeiro@gmail.com
}

\begin{abstract}
This study proposed to estimate the prevalence of behavioral disorders and associated factors in adolescents (11-15 years), using a cross-sectional design $(n=1,145)$. Subjects answered a self-administered questionnaire. Behavioral disorder was assessed with the Mini International Neuropsychiatric Interview (MINI). The study analyzed disorders in relation to gender, age, socioeconomic status, schooling, failure in school, religion, smoking, sedentary lifestyle, alcohol consumption, drug use, depression, and bullying (as victim). Ordinal regression was used for the statistical analysis, with a hierarchical model for the outcome. An estimated $29.2 \%$ of the sample presented behavioral disorders. In the multivariate analysis, the odds ratio for a male adolescent to present one additional point on the behavioral disorder scale was 2.04 (95\%CI: 1.53-2.71). Alcohol consumption, drug use, and suffering bullying were associated with higher scores on the behavioral disorder scale. The findings also showed that the factors associated with behavioral disorder showed a strong interrelationship between health behaviors in adolescence.
\end{abstract}

Adolescent Behavior; Conduct Disorder; Adolescent

\author{
Ana Laura Sica Cruzeiro ${ }^{1}$ \\ Ricardo Azevedo da Silva ${ }^{1}$ \\ Bernardo Lessa Horta ${ }^{2}$ \\ Luciano Dias de Mattos Souza 1 \\ Augusto Duarte Faria 1 \\ Ricardo Tavares Pinheiro 1 \\ Inessa de Oliveira Silveira 1 \\ Cecília Dieguez Ferreira 1
}

\section{Introdução}

Os transtornos da conduta abrangem os comportamentos de risco que podem comprometer a saúde física e mental do adolescente 1 , como o uso de álcool, o uso de drogas e violência. Estão entre os transtornos mais comumente encontrados na infância e adolescência ${ }^{2,3,4}$ e são padrões de comportamentos repetitivos e persistentes, no qual são violados direitos básicos dos outros, normas ou regras sociais 5 , iniciando comportamentos agressivos e reagindo agressivamente a outras pessoas 6,7 .

Segundo o Diagnostic and Statistical Manual of Mental Disorders, $4^{\text {th }}$ Edition (DSM-IV-TR) 6, o transtorno da conduta, classificado nos "transtornos de comportamentos disruptivos da infância e da adolescência", pode ser dividido em conduta agressiva que causa ou ameaça danos físicos a outras pessoas ou a animais; conduta não-agressiva que causa perdas ou danos a propriedades; defraudação e/ou furto; e sérias violações de regras.

Nesta desordem psicológica, os comportamentos inadequados de menor gravidade como: mentir, enganar, faltar aulas sem justificativa e furtar objetos de pouco valor, costumam anteceder comportamentos mais graves como brigas com uso de armas, arrombamentos e assaltos 8 . A persistência dos comportamentos que caracterizam o transtorno pode conduzir a um diagnósti- 
co de transtorno de personalidade anti-social na idade adulta $6,8,9,10,11,12,13$.

A prevalência de transtorno da conduta varia bastante entre os países em que foram realizados os estudos, desde 5,5\% no Canadá 14,15 até 30,4\% no Peru ${ }^{4}$. Na maioria dos estudos os meninos apresentam um risco maior de apresentarem transtornos de conduta 8,10,14,15,16. Além disso, é mais freqüente em adolescentes mais velhos, independente do sexo 8 .

Os fatores mais comumente associados aos transtornos de conduta são: sexo 4,7, nível sócioeconômico 14,15,17,18,19,20, uso de bebida alcoólica 4,21,22,23,24,25, uso/abuso de drogas ilícitas 26,27,28, bullying 29 e depressão 30,31. Não existem estudos brasileiros sobre a freqüência e os fatores comportamentais associados ao problema. Nesse sentido, faz-se necessário estimar a prevalência e os fatores comportamentais associados ao transtorno da conduta em adolescentes para que se possa ajudar no estabelecimento de estratégias preventivas.

\section{Metodologia}

Realizou-se um estudo transversal em uma amostra representativa com adolescentes de 11 a 15 anos na cidade de Pelotas, Rio Grande do Sul, Brasil, em 2006. Esta investigação fez parte de uma ampla pesquisa sobre comportamentos de saúde na adolescência, realizada pelo Programa de Pós-graduação em Saúde e Comportamento, da Escola de Pscologia, da Universidade Católica de Pelotas (UCPel). O projeto foi aprovado pelo Comitê de Ética em Pesquisa da Universidade Federal do Rio Grande do Sul (UFRGS).

A amostragem foi realizada em múltiplos estágios. A partir dos 448 setores censitários da zona urbana da cidade foram sorteados 79 setores. Em cada um desses setores selecionados, sortearam-se um quarteirão e uma esquina como ponto inicial a partir de onde iniciou-se a visita sistemática de todas residências.

A partir da estimativa de prevalência encontrada na revisão, de um nível de $95 \%$ de confiança e de um poder estatístico de $80 \%$ e erro amostral de $1,5 \%$, calculou-se o tamanho da amostra necessário para identificar a prevalência e avaliar a associação entre o transtorno da conduta e comportamentos em saúde na adolescência, assim seria necessário acompanhar uma amostra de 856 adolescentes. Ao serem acrescentados mais $30 \%$ para perdas e para controle de fator de confusão, conseqüentemente teriam que ser acompanhados 1.112 adolescentes.

Após a obtenção do consentimento por escrito dos pais ou responsável pelo adolescente, o jo- vem respondeu a um questionário auto-aplicado e sigiloso com questões sobre sexo, idade, nível sócio-econômico, escolaridade, religiosidade, atividades físicas na escola e de lazer, entreterimento, consumo de álcool, tabagismo, uso de drogas ilícitas, relacionamento sexual, depressão, comportamentos agressivos e sofrer bullying.

Bullying compreende todas as formas de atitudes agressivas, intencionais e repetidas, que ocorrem sem motivações evidentes, adotadas por um ou mais indivíduo contra outro(s), causando dor, angústia e humilhação, e executadas dentro de uma relação desigual de poder. O estudo avaliou os adolescentes terem sofrido bullying.

Para avaliação do transtorno da conduta foi utilizado, na forma auto-aplicada, o módulo do Mini International Neuropsychiatric Interview (MINI) ${ }^{32}$ que investiga esta patologia, um instrumento compatível com os critérios do Diagnostic and Statistical Manual of Mental Disorders, 3 rd Edition, Revised (4th Edition) (DSM-III-R/IV) e da Classificação Internacional de Doenças, 10a revisão (CID-10), investigando as seguintes variáveis: ter freqüentemente passado a noite na rua, desacompanhado, sem autorização dos pais; ter freqüentemente mentido, passado a perna/enganado os outros; ter roubado alguma coisa; ter ameaçado bater em outras pessoas; ter destruído ou incendiado coisas de propósito; e ter machucado animais ou pessoas de propósito.

Para a estimativa de depressão foi utilizado o Inventário de Depressão Infantil (CDI) validado para a cidade brasileira de João Pessoa, no Estado da Paraíba, no qual constam vinte itens ${ }^{33}$. Com a soma dos escores de todos os itens, obteve-se o escore total para cada adolescente.

Os dados coletados foram digitados no programa Epi Info 6.0 (Centers for Disease Control and Prevention, Atlanta, Estados Unidos), com checagem automática da consistência. Primeiramente, foi calculada a prevalência do transtorno, utilizando o ponto de corte para esta estimativa, estabelecido no instrumento, duas ou mais respostas positivas para os comportamentos investigados. Logo após, utilizou-se a regressão ordinal na análise estatística com o somatório de respostas positivas de cada adolescente, no Stata 9.0 (Stata Corp., College Station, Estados Unidos), quando o modelo de odds proporcional foi empregado para estimar o risco de aumentar em um ponto na escala. Os riscos brutos foram testados para o requisito de proporcionalidade do odds, usando o teste de Brant. Todas as análises controlaram para o efeito do delineamento, uma vez que se utilizou uma amostragem em múltiplos estágios.

$\mathrm{Na}$ análise multivariada, usou-se um modelo hierárquico, onde cada bloco de variáveis de um 
determinado nível foi incluído, e as variáveis com um valor $\mathrm{p} \leq 0,20$ no teste de razões de verossimelhança permaneceram no modelo. Nesse tipo de modelo, as variáveis situadas em um nível hierárquico superior ao da variável em questão são consideradas como potenciais confundidores da relação entre essa variável e o desfecho em estudo, enquanto que as variáveis em níveis inferiores são consideradas como potenciais mediadores da associação 34 . As variáveis selecionadas em um determinado nível permaneceram nos modelos subseqüentes e foram consideradas como fatores associados com transtorno da conduta mesmo que, com a inclusão de variáveis hierarquicamente inferiores, tivessem perdido sua significância.

O modelo hierárquico foi composto, de acordo com a revisão de literatura 4,14,22,26,27,30, da seguinte maneira: no primeiro nível, as variáveis sócio-demográficas (sexo, idade e nível sócioeconômico); no segundo, as variáveis comportamentais (escolaridade do adolescente, reprovação na escola, ter uma religião, freqüentar culto ou missa da religião a que pertence, sedentarismo, consumo de bebida alcoólica no último mês, tomar porre no último mês, uso de cigarro no último mês e uso de drogas no último mês); e, no terceiro nível, as variáveis psicossociais (depressão e sofrer bullying).

\section{Resultados}

Dos 1.265 adolescentes localizados, 120 (9,9\%) se recusaram a participar do estudo ou foram considerados perdas, totalizando 1.145 adolescentes entrevistados com idades de 11 a 15 anos.

A amostra de adolescentes entrevistados estava dividida de forma similar em relação ao sexo, $51,7 \%$ do sexo feminino e $48,3 \%$ do sexo masculino. Assim também esteve dividida nas idades, com um percentual em torno de $20 \%$ para cada faixa etária. Cabe ressaltar o grande percentual de reprovação na escola (44\%). Quanto à religiosidade, $62,7 \%$ tinham religião e $45,8 \%$ freqüentavam o culto ou a missa de sua religião.

Ao avaliar-se a prevalência de transtorno da conduta, segundo os critérios do instrumento utilizado, encontramos que $29,2 \%$ relataram dois ou mais dos comportamentos avaliados.

Na análise bivariada (Tabela 1), os adolescentes do sexo masculino tiveram 1,91 vezes (IC95\%: 1,50-2,43) mais chance de apresentar um comportamento a mais do que as meninas. Em relação à faixa etária, os adolescentes de 13 anos (odds ratio - OR = 1,50; IC95\%: 1,05-2,16) e 14 anos $(\mathrm{OR}=1,46$; IC95\%: 1,02-2,09) mostraram maior chance de apresentar mais comportamentos relacionados com o transtorno da conduta.
Tabela 1

Odds ratio (OR) para escore de transtorno da conduta (regressão ordinal) de acordo com variáveis sócio-econômicas e demográficas. Pelotas, Rio Grande do Sul, Brasil, 2006.

\begin{tabular}{|c|c|c|}
\hline Variável & $\mathrm{n}$ & OR (IC95\%) \\
\hline \multicolumn{3}{|l|}{ Sexo } \\
\hline Masculino & 540 & $1,91(1,50-2,43)$ \\
\hline Feminino & 557 & Referência \\
\hline \multicolumn{3}{|l|}{ Idade (anos) } \\
\hline 11 & 227 & Referência \\
\hline 12 & 235 & $0,84(0,58-1,21)$ \\
\hline 13 & 224 & $1,50(1,05-2,16)$ \\
\hline 14 & 230 & $1,46(1,02-2,09)$ \\
\hline 15 & 207 & $1,17(0,84-1,64)$ \\
\hline \multicolumn{3}{|l|}{ Nível sócio-econômico } \\
\hline Baixo & 310 & $1,65(1,10-2,46)$ \\
\hline Médio & 265 & $1,07(0,73-1,56)$ \\
\hline Alto & 292 & Referência \\
\hline \multicolumn{3}{|c|}{ Escolaridade do adolescente (anos de estudo) } \\
\hline Até 4 & 167 & Referência \\
\hline 5 a 7 & 682 & $1,13(0,76-1,70)$ \\
\hline 8 ou mais & 231 & $1,11(0,72-1,73)$ \\
\hline \multicolumn{3}{|l|}{ Reprovação na escola } \\
\hline Nenhuma vez & 588 & Referência \\
\hline Uma vez & 260 & $1,31(1,02-1,70)$ \\
\hline Duas ou mais vezes & 202 & $1,53(1,14-2,07)$ \\
\hline \multicolumn{3}{|l|}{ Religião } \\
\hline Sim & 688 & Referência \\
\hline Não & 410 & $1,27(1,01-1,59)$ \\
\hline \multicolumn{3}{|c|}{ Freqüência ao culto ou à missa } \\
\hline Sim & 501 & Referência \\
\hline Não & 594 & $1,41(1,11-1,78)$ \\
\hline \multicolumn{3}{|l|}{ Sedentarismo } \\
\hline Sedentário & 152 & Referência \\
\hline Ativo & 596 & $1,44(1,04-2,00)$ \\
\hline \multicolumn{3}{|c|}{ Consumir bebida alcoólica (último mês) } \\
\hline Sim & 249 & $2,64(2,00-3,49)$ \\
\hline Não & 896 & Referência \\
\hline \multicolumn{3}{|l|}{ Tomar porre (último mês) } \\
\hline Sim & 48 & $3,03(1,80-5,10)$ \\
\hline Não & 1.097 & Referência \\
\hline \multicolumn{3}{|c|}{ Uso de cigarro (último mês) } \\
\hline Sim & 80 & $2,28(1,32-3,94)$ \\
\hline Não & 1.065 & Referência \\
\hline \multicolumn{3}{|c|}{ Uso de drogas (último mês) } \\
\hline Sim & 39 & $5,93(3,32-10,60)$ \\
\hline Não & 1.006 & Referência \\
\hline \multicolumn{3}{|l|}{ Depressão } \\
\hline Sim & 20 & $5,14(1,05-25,19)$ \\
\hline Não & 931 & Referência \\
\hline \multicolumn{3}{|l|}{ Bullying } \\
\hline Sim & 244 & $2,14(1,55-2,97)$ \\
\hline Não & 523 & Referência \\
\hline
\end{tabular}


Em relação ao nível sócio-econômico os adolescentes de classe mais baixa apresentaram uma chance 1,65 vezes maior (IC95\%: 1,10-2,46). Além do mais, ter reprovado de ano na escola esteve associado com aumento no número de comportamentos relacionados ao transtorno da conduta (Tabela 1).

Pertencer a uma religião $(\mathrm{OR}=1,27$; IC95\%: 1,01-1,59) e freqüentar o culto ou missa dessa religião (OR = 1,41; IC95\%: 1,11-1,78) se mostraram um fator protetor para desenvolvimento de transtorno da conduta.

Entre os comportamentos de saúde na adolescência examinados, o sedentarismo (OR = 1,44; IC95\%: 1,04-2,00), o consumo de bebidas alcóolicas no último mês (OR = 2,64; IC95\%: 2,00$3,49)$, tomar porre no último mês $(\mathrm{OR}=3,03$; IC95\%: 1,80-5,10), o uso de cigarro no último mês $(\mathrm{OR}=2,28$; IC95\%: 1,32-3,94) e o uso de drogas no último mês (OR = 5,93; IC95\%: 3,32-10,60) estiveram associados a um maior número de comportamentos relacionados com o transtorno da conduta.

As duas variáveis psicossociais examinadas: depressão (OR = 5,14; IC95\%: 1,05-25,19) e sofrer bullying (OR = 2,14; IC95\%: 1,55-2,97) também mostraram-se associadas (Tabela 1).

Após o ajuste realizado através da técnica de regressão ordinal, segundo o modelo hierarqui- zado, para controlar possíveis fatores de confusão e estabelecer possíveis fatores mediadores, mantiveram uma associação significativa as variáveis sexo, idade dos adolescentes e nível sócio-econômico (Figura 1) do primeiro nível hierárquico. A OR para o sexo masculino aumentou de 1,91 para 2,04 (IC95\%: 1,53-2,71). As idades de 13 anos (OR = 2,07; IC95\%: 1,34-3,21) e 14 anos $(\mathrm{OR}=1,76$; IC95\%: 1,15-2,71) mantiveram-se associadas ao transtorno, bem como o nível sócio-econômico baixo (OR = 1,89; IC95\%: 1,27-2,80).

No segundo nível as variáveis que permaneceram associadas foram consumo de bebida alcoólica (OR = 2,36; IC95\%: 1,68-3,31) e uso de drogas no último mês (OR = 3,11; IC95\%: 1,645,91).

Por fim, no terceiro nível hierárquico, apenas sofrer bullying manteve-se associado $(\mathrm{OR}=2,02$; IC95\%: 1,26-3,26).

\section{Discussão}

Inicialmente, é importante comentar sobre as dificuldades terminológicas enfrentadas diante das nomenclaturas encontradas. Na literatura, o termo transtorno da conduta, geralmente, equipara-se a comportamento anti-social. No entanto, usa-se o termo comportamento anti-

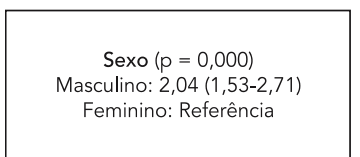

$\begin{aligned} & \operatorname{Sexo}(p=0,000) \\ & \text { Fasculino: } 2,04(1,53-2,71)\end{aligned}$
Idade em anos $(p=0,008)$

11: Referência

12: $0,93(0,61-1,43)$

13: $2,07(1,34-3,21)$

14: $1,76(1,15-2,71)$

$15: 1,37(0,90-2,09)$
Nível sócio-econômico $(p=0,003)$

Baixo: $1,89(1,27-2,80)$

Médio: $1,20(0,83-1,72)$

Alto: Referência
Consumo bebida alcoólica no último mês $(p=0,001)$
Sim: $2,36(1,68-3,31)$
Não: Referência
Uso de drogas no último mês $(p=0,001)$

Sim: 3,11 $(1,64-5,91)$

Não: Referência 
social, para referir-se a diversas características comportamentais de diversos tipos de transtornos mentais 11 que são englobados por tal terminologia, como: transtorno da conduta, transtorno desafiador opositivo e transtorno de personalidade anti-social. O termo comportamento anti-social não necessita de um único diagnóstico clínico, enquanto que o transtorno da conduta apresenta um quadro de sintomas característicos da patologia.

Este é o primeiro estudo realizado no Brasil que mostra a prevalência de transtorno da conduta. Existem poucos estudos no mundo que mostram esta prevalência em adolescentes, no Brasil só foi encontrado um estudo sobre comportamentos anti-sociais, em que não é descrita a prevalência bruta, apresentando apenas a prevalência de comportamentos violentos e comportamentos não-violentos de forma separada 35 .

Os estudos encontrados apresentam prevalências muito variadas: no Canadá 14,15, uma investigação com instrumento baseado nos critérios do DSM-III respondido pelo adolescente, pais e professores, mostrou que 5,5\% da população de 4 a 16 anos apresentaram esta psicopatologia, enquanto que no Peru 4 , o transtorno foi encontrado em $30,4 \%$ da população de 421 adolescentes de 12 a 17 anos de idade, avaliados através do Cuestionario de Salud Mental. As diferenças nos valores, do Brasil e Peru em relação ao Canadá, provavelmente são decorrentes da divergência de instrumentos utilizados nas coletas de dados e nas diferenças sócio-econômicas e culturais dos locais investigados.

Em relação ao sexo, os estudos 3,4 apontam resultados no mesmo sentido, o sexo masculino apresenta maiores chances de desenvolver transtorno da conduta comparado com o sexo feminino, corroborando os resultados encontrados em Pelotas. Essa associação provavelmente se deve a algumas diferenças que caracterizam o sexo masculino e o sexo feminino. Em estudo realizado no Brasil com escolares, o sexo masculino esteve significativamente associado à vivência $\mathrm{e}$ expressão da agressividade 36 .

Assim como neste trabalho, alguns estudos 14,15,17,18,19,20 têm mostrado que o baixo nível sócio-econômico é um fator de risco para muitos comportamentos perigosos na adolescência.

No já citado trabalho realizado no Peru ${ }^{4} \mathrm{com}$ adolescentes, os dados encontrados mostraram que, como no presente estudo, o abuso de bebida alcoólica está significativamente associado com o transtorno da conduta $(\mathrm{OR}=6,91 ; \mathrm{p}<0,001)$. Clark et al. 23, em estudo realizado com adolescentes de 14 a 18 anos, também mostraram esta associação entre os usuários de álcool que estavam em tratamento (89\%). É possível levantar a hipótese de o álcool, por suas características depressoras do sistema nervoso central, facilitar o aparecimento de comportamentos disruptivos.

A comorbidade entre o uso/abuso de drogas e transtorno da conduta é corroborada por diversos autores 26,27,28; um estudo em Taiwan 31 sobre transtorno por uso de substâncias mostra que a comorbidade apresenta um OR de 9,5. Esta relação poderá levar à violência e à criminalidade 37,38 , pois muitos estudos mostram que o uso de drogas é preditor da violência/criminalidade e que o transtorno da conduta e o abuso de drogas estão presentes em jovens encarcerados 39 .

Assim como em Pelotas, um estudo em 25 países da Europa, Health Behaviour in School-aged Children Study (HBSC Study), mostrou que sofrer bullying está significativamente associado a altas taxas de problemas de comportamento ${ }^{29}$. Este estudo europeu também mostrou a associação com o pobre ajustamento emocional, que pode levar ao aumento da agressividade do jovem possibilitando o aparecimento do transtorno da conduta 29. É plausível que os adolescentes que sofreram violência tornem-se violentos.

Alguns estudos mostram o transtorno da conduta como fator associado à depressão 30,31, mas a presente investigação, após o ajuste ao modelo hierárquico, não encontrou associações significativas entre estas duas patologias. Provavelmente devido à utilização de um modelo hierarquizado para o controle de variáveis no presente estudo, que permite maior precisão nos resultados, e a perda de poder dado a baixa prevalência de depressão na amostra estudada. Esta baixa prevalência pode estar relacionada ao instrumento utilizado, que avalia somente alguns sintomas de depressão.

Em relação às características do estudo, cabe salientar que por ser transversal de base populacional e com taxa de resposta de $92,4 \%$ reduz-se a possibilidade de ocorrência de viés de seleção. Ademais, a amostra do estudo representa a população de Pelotas, de acordo com o levantamento do Departamento de Informática do SUS (DATASUS; http://www.datasus.gov.br) de 2006, quando o número de homens e mulheres, na faixa etária dos 10 aos 14 anos, era respectivamente 14.856 e 14.586. Por outro lado, não ter avaliado as questões sociais e familiares possivelmente associadas ao transtorno da conduta são limitações desta investigação.

O estudo foi realizado com adolescentes de 11 a 15 anos de idade, porque existem poucos estudos com jovens desta faixa etária e os sintomas da patologia que aparecem na adolescência inicial geralmente persistem por mais tempo e podem chegar a um transtorno de personalidade anti-social 6,8,9,10,11,12,13. Além disso, quanto mais 
precoce a identificação das características relacionadas à psicopatologia e, assim, as primeiras intervenções ocorrerem, maiores as chances de haver uma inibição do desenvolvimento da doença ${ }^{7}$.

Nesse contexto, sexo e nível sócio-econômico estão significativamente associados à patologia. Além disso, os demais fatores associados ao transtorno da conduta apontam para a forte interrelação entre comportamentos de saúde na adolescência. Estratégias preventivas com relação à precocidade do abuso de substâncias e sofrer bullying podem evitar o agravamento do quadro, pois são comportamentos preditores do transtorno da conduta.

\section{Resumo}

Este trabalho procurou estimar a prevalência e os fatores associados ao transtorno da conduta em adolescentes com idade entre 11 e 15 anos. Realizou-se um estudo transversal com 1.145 adolescentes na cidade de Pelotas, Rio Grande do Sul, Brasil. Os adolescentes responderam a um questionário auto-aplicado. $O$ transtorno da conduta foi avaliado através do Mini International Neuropsychiatric Interview (MINI). Foi investigada a associação desta psicopatologia com sexo, idade, nível sócio-econômico, escolaridade do adolescente, reprovação na escola, religião, sedentarismo, consumo de álcool, tabagismo, uso de drogas, depressão e sofrer bullying. A regressão ordinal foi usada para a análise estatística. Estima-se que, entre os entrevistados, 29,2\% tenham transtorno da conduta. Na análise multivariada, a chance de um adolescente apresentar um ponto a mais na escala que avalia transtorno da conduta foi 2,04 (IC95\%: 1,53-2,71) vezes maior no sexo masculino. O consumo de bebida alcoólica, o uso de drogas e sofrer bullying estiveram associados com maior pontuação na escala de transtornos de conduta. Os resultados também mostraram que os fatores associados ao transtorno da conduta apontam para a forte interrelação entre comportamentos de saúde na adolescência.

Comportamento do Adolescente; Transtorno da Conduta; Adolescente
$\mathrm{Na}$ literatura, existem estudos que apontam a existência de estratégias de tratamentos cognitivo-comportamentais, com jovens 10 e familiares 7 , eficazes para a redução da sintomatologia do transtorno da conduta.

Tendo em vista os resultados apontados, uma contribuição significativa do presente estudo é fornecer subsídios para iniciar um programa de diagnósticos precoces. Pois, crianças e adolescentes com transtornos de comportamento tendem a permanecer com estes transtornos na idade adulta, e estes tendem a criar filhos com transtornos de comportamentos 40 , estabelecendo-se um ciclo difícil para a sociedade.

\section{Colaboradores}

A. L. S. Cruzeiro, R. A. Silva, B. L. Horta e R. T. Pinheiro conceberam o presente artigo B. L. Horta colaborou e revisou a análise estatística. A. L. S. Cruzeiro, L. D. M. Souza e A. D. Faria supervisionaram o trabalho de campo e a ação dos entrevistadores, além da elaboração do texto final. I. O. Silveira e C. D. Ferreira participaram da dupla digitação dos dados do estudo e da revisão bibliográfica para o artigo. R. A. Silva e R. T. Pinheiro realizaram a revisão final. 


\section{Referências}

1. Feijó RB, Oliveira EA. Comportamento de risco na adolescência. J Pediatr (Rio J) 2001; 77 Suppl 2: S125-34.

2. Vloet TD, Herpertz S, Herpertz-Dahlmann B. Aetiology and life-course of conduct disorder in childhood: risk factors for the development of an antisocial personality disorders. Z Kinder Jugendpsychiatr Psychother 2006; 34:101-14.

3. Simonoff E, Elander J, Holmshaw J, Pickles A, Murray R, Rutter M. Predictors of antisocial personality: continuities from childhood to adult life. Br J Psychiatry 2004; 184:118-27.

4. Perales A, Sogi C. Perspectiva sociocultural de las conductas disociales em adolescentes. Acta Psiquiatr Psicol Am Lat 1998; 44:29-39.

5. Kaplan HI, Sadock BJ, Grebb JA. Compêndio de psiquiatria: ciências do comportamento e psiquiatria clínica. Porto Alegre: Editora Artes Médicas; 1997.

6. American Psychiatric Association. Diagnostic and statistical manual of mental disorders. $4^{\text {th }} \mathrm{Ed}$ Washington DC: American Psychiatric Association; 1994.

7. Marinho ML, Caballo VE. Comportamento antisocial infantil e seu impacto para a competência social. Psicol Saúde Doenças 2002; 3:141-7.

8. Bordin IAS, Offord DR. Transtorno da conduta e comportamento anti-social. Rev Bras Psiquiatr 2000; 22(Suppl II):12-5.

9. Farrington DP. Childhood origins of teenage antisocial behavior and adult social dysfunction. J R Soc Med 1993; 86:13-7.

10. Moffitt TE, Caspi A. Childhood predictors differentiate life-course persistent and adolescence-limited antisocial pathways among males and females. Dev Psychopathol 2001; 13:355-75.

11. Pacheco J, Alvarenga P, Reppold C, Piccinini CA, Hultz CS. Estabilidade do comportamento anti-social na transição da infância para a adolescência: uma perspectiva desenvolvimentista. Psicol Reflex Crit 2005; 18:55-61.

12. Soderstrom H, Sjodin A, Carlstedt A, Forsman A. Adult psychopathic personality with childhoodonset hyperactivity and conduct disorder: a central problem constellation in forensic psychiatry. Psychiatry Res 2004; 121:271-80.

13. Goldstein RB, Grant BF, Ruan JW, Smith SM, Saha TD. Antisocial personality disorder with childhood- vs. adolescence-onset conduct disorder: results from the National Epidemiologic Survey on Alcohol and Related Conditions. J Nerv Ment Dis 2006;194:667-75

14. Offord DR, Boyle MH, Fleming JE, Blum HM, Grant NIA. Ontario Child Health Study: summary of selected results. Can J Psychiatry 1989; 34:483-91.

15. Offord DR, Boyle MH, Racine Y. Ontario Child Health Study: correlates of disorder. J Am Acad Child Adolesc Psychiatry 1989; 28:856-60.

16. Ma HK. The relation of gender-role classifications to the prosocial and antisocial behavior of chinese adolescents. J Genet Psychol 2005; 166:189-201.
17. Pineda DA, Kamphaus RW, Restrepo MA, Puerta IC, Arango CP, Lopera FJ, et al. Screening for conduct disorder in an adolescent male sample from Colombia. Transcult Psychiatry 2006; 43:362-82.

18. Lahey BB, Loeber R, Burke JD, Applegate B. Predicting Future antisocial personality disorder in males from a clinical assessment in childhood. J Consult Clin Psychol 2005; 73:389-99.

19. Lacourse E, Nagin DS, Vitaro F, Côté S, Arseneault L, Tremblay RE. Prediction of early-onset deviant peer group affiliation - a 12-year longitudinal study. Arch Gen Psychiatry 2006; 63:562-8.

20. Bassarath L. Conduct disorder: a biopsychosocial review. Can J Psychiatry 2001; 46:609-16.

21. Murray J, Farrington DP. Parental imprisonment: effects on boys' antisocial behaviour and delinquency through the life-course. J Child Psychol Psychiatry 2005; 46:1269-78

22. Clark DB, Bukstein OG. Psychopathology in adolescent alcohol abuse and dependence. Alcohol Health Res World 1998; 22:117-21.

23. Clark DB, Pollock N, Bukstein OG, Mezzich AC Bromberger JT, Donovan JE. Gender and comorbid psychopathology in adolescents with alcohol dependence. J Am Acad Child Adolesc Psychiatry 1997; 36:1195-203.

24. Brown SA, Gleghorn A, Schuckit MA, Myers MG, Mott MA. Conduct disorder among adolescent alcohol and drug abusers. J Stud Alcohol 1996; 57:314-24.

25. Couwenbergh C, Brink W, Zwart K, Vreugdenhil C, Wijngaarden-Cremers P, Gaag RJ. Comorbid psychopathology in adolescents and young adults treated for substance use disorders: a review. Eur Child Adolesc Psychiatry 2006; 15:319-28.

26. Chong MY, Chan KW, Cheng ATA. Substance use disorders among adolescents in Taiwan: prevalence, sociodemographic correlates and psychiatric co-morbidity. Psychol Med 1999; 29:1387-96.

27. Armstrong TD, Costello JE. Community studies on adolescent substance use, abuse, or dependence and psychiatric comorbidity. J Consult Clin Psychol 2002; 70:1224-39.

28. Costello EJ, Mustillo S, Erkanli A, Keeler G, Angold A. Prevalence and development psychiatric disorders in childhood and adolescence. Arch Gen Psychiatry 2003; 60:837-44.

29. Nansel TR, Craig W, Overpeck MD, Saluja G, Ruan WJ; Health Behaviour in School-aged Children Bullying Analyses Working Group. Cross-national consistency in the relationship between bullying behaviors and psychosocial adjustment. Arch Pediatr Adolesc Med 2004; 158:730-6.

30. Zoccolillo M. Co-occurrence of conduct disorder and its adult outcomes with depressive and anxiety disorders: a review. J Am Acad Child Adolesc Psychiatry 1992; 31:547-57.

31. Kovacs M, Pollock M. Bipolar disorder and comorbid conduct disorder in childhood and adolescence. J Am Acad Child Adolesc Psychiatry 1995; 34:715-23. 
32. Amorim P. Mini International Neuropsichyatric Interview (MINI): validação de entrevista breve para diagnóstico de transtornos mentais. Rev Bras Psiquiatr 2000; 22:106-15.

33. Gouveia VV, Barbosa GA, Almeida HJF, Gaião AA. Inventário de depressão infantil - CDI: estudo de adaptação com Escolares de João Pessoa. J Bras Psiquiatr $1995 ; 44: 345-9$

34. Victora CG, Huttly SR, Fuchs SC, Olinto MTA. The role of conceptual frameworks in epidemiological analysis: a hierarchical approach. Int J Epidemiol 1997; 26:224-7.

35. Bordin IAS. Fatores de risco para comportamento anti-social na adolescência e início da vida adulta [Tese de Doutorado]. São Paulo: Escola Paulista de Medicina, Universidade Federal de São Paulo; 1996.

36. Guimarães NM, Pasian SR. Agressividade na adolescência: experiência e expressão da raiva. Psicol Estud 2006; 11:89-97.
37. Dawkins MP. Drug use and violent crime among adolescents. Adolescence 1997; 32:395-405.

38. Herrenkohl TI, Maguin E, Hill KG, Hawkins JD, Abbott RD, Catalano RF. Developmental risk factors for youth violence. J Adolesc Health 2000; 26:176-86.

39. Copur M, Turkcan A, Erdogmus M. Substance abuse, conduct disorder and crime: Assessment in a juvenile detention house in Istanbul, Turkey. Psychiatry Clin Neurosci 2005; 59:151-4.

40. Farrington DP. The Twelfth Jack Tizard Memorial Lecture. The development of offending and antisocial behaviour from childhood: key findings from the Cambridge Study in Delinquent Development. J Child Psychol Psychiatry 1995; 36:929-64.

Recebido em 26/Jul/2007

Versão final reapresentada em 22/Jan/2008 Aprovado em 20/Fev/2008 